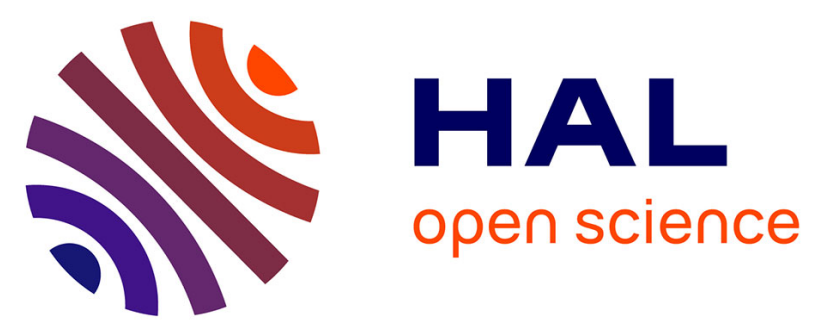

\title{
Asynchronous and decoupled control of the position and the stiffness of a spatial RCM tensegrity mechanism for needle manipulation
}

Jurado Realpe, Guillaume Aiche, Salih Abdelaziz, Philippe Poignet

\section{- To cite this version:}

Jurado Realpe, Guillaume Aiche, Salih Abdelaziz, Philippe Poignet. Asynchronous and decoupled control of the position and the stiffness of a spatial RCM tensegrity mechanism for needle manipulation. ICRA 2020 - 37th IEEE International Conference on Robotics and Automation, May 2020, Paris (Virtual), France. pp.3882-3888, 10.1109/ICRA40945.2020.9197507 . lirmm-03140522

\section{HAL Id: lirmm-03140522 \\ https://hal-lirmm.ccsd.cnrs.fr/lirmm-03140522}

Submitted on 12 Feb 2021

HAL is a multi-disciplinary open access archive for the deposit and dissemination of scientific research documents, whether they are published or not. The documents may come from teaching and research institutions in France or abroad, or from public or private research centers.
L'archive ouverte pluridisciplinaire HAL, est destinée au dépôt et à la diffusion de documents scientifiques de niveau recherche, publiés ou non, émanant des établissements d'enseignement et de recherche français ou étrangers, des laboratoires publics ou privés. 


\title{
Asynchronous and decoupled control of the position and the stiffness of a spatial RCM tensegrity mechanism for needle manipulation.*
}

\author{
JR Jurado Realpe ${ }^{1}$, G. Aiche ${ }^{1}$, S. Abdelaziz ${ }^{1}$ and P. Poignet ${ }^{1}$.
}

\begin{abstract}
This paper introduces a 2-DOF spatial remote center of motion (RCM) tensegrity mechanism, based on a double parallelogram system, dedicated for percutaneous needle insertion. The originality of this mechanism is its ability to be reconfigured and its capacity to perform a decoupled modulation of its stiffness in an asynchronous way. To do so, an analytical stiffness model of the robot is established, and a control methodology is proposed. A prototype of the robot is developed and assessed experimentally. The position tracking is evaluated using a 6-DOF magnetic tracker sensor showing a root mean square error less than $0.8^{\circ}$ in both directions of the needle guide.
\end{abstract}

\section{INTRODUCTION}

Tensegrity systems are increasingly attracting the interest of roboticians for their large workspace and their ability to vary its stiffness. They were introduced for the first time in 1962 by Fuller [1]. Tensegrity systems can be defined as an assembly of a set of elements subjected to compression forces and others to tensile forces, and this for all configuration of the system. The set of the compressive elements interacts with the set of tensile components to define a stable structure [2]. The fact of using tensile elements allows the introduction of cables and springs to lighten the structure. In the literature, tensegrity systems have been considered as structures [3], [4], mobile robots [5] or deployable systems [6], [7].

Research on control for tensegrity systems was initiated in the mid-1990s, with efforts dedicated to establish dynamic model of these structures [8]. Today, most of the control contributions of these systems are related to disturbance rejection and motion control. Disturbance rejection refers to structures subject to deformation and vibration due in particular to thermal expansion or atmospheric fluctuations such as the wind or seismic disturbances. Motion control addresses the robots whose architecture is based on tensegrity, in particular mobile robots and deployable mechanisms.

For mobile robot and deployable mechanisms, trajectory tracking is not considered as an absolute priority. Indeed, the principal focus of these systems is the production of gait, in the case of mobile robot [5] and the deployment ability for deployable systems. For these latter, several open-loop control approaches have been introduced [6], [7] under the assumption that reconfiguration is performed in quasi-static

\footnotetext{
*This work was supported by French state funds managed by the ANR within the Investissements d'Avenir programme (Robotex ANR-10-EQPX44, Labex CAMI - ANR- 11-LABX-0004)

1 The authors are with the LIRMM, University of Montpellier, CNRS, France. [juradareal, abdelaziz, poignet] Qlirmm.fr.
}

while neglecting dynamic effects. Besides, dynamic has also been taken into account in the development of open-loop control laws, considering in particular the dynamic model as a constraint in the optimization procedure to minimize the execution time [9] and the energy required for deployment [10].The problem of rapid tensegrity control has been also addressed in [11] by using infinitesimal mechanisms and non-linear feedback control.

For the locomotion control of mobile tensegrity robots, open-loop control strategies based on genetic and evolutionary algorithms [5], [12], [13] and neural networks [14] have been developed to overcome complex dynamic modeling and precise knowledge of the system's physical environment. Other strategies, this time in a closed loop, have been proposed in order to minimize vibrations during the movement of the structure [15] by using on the one hand a $H_{\infty}$ controller based on a linearization of the dynamic model and on the other hand to compensate for inaccuracies of the dynamic model [16] by using a non-linear decoupling control.

Although these control strategies are of interest to improve the performance of tensegrity systems in terms of disturbance rejection, locomotion and deployment capacity, we are interested here in controlling these systems from a very different perspective. Indeed, our motivation is to control the reconfiguration and trajectory tracking of tensegrity systems, considered in this work as manipulators, while explicitly managing their stiffness. This paper is the continuation of the work of [17] where a proof of concept of this motivation has been demonstrated through a planar 1-degree of freedom (DOF) mechanism. In this work, this proof of feasibility is evaluated through a new spatial 2-DOF tensegrity mechanism, where analytical stiffness model of the mechanism is derived. Decoupling to control the position or the stiffness along one of the mobilities without affecting the other mobility is also addressed.

This paper is organized as follow. In the second section, the requirements and an overview of the mechanism is presented. Section 3 introduces the direct and inverse kinematic models. In section 4, the static and the stiffness models are derived. Section 5 focuses on the device control of the prototype where results on position tracking and stiffness modulation are discussed. Conclusions and future work are developed in section 6 . 


\section{REQUIREMENTS AND SYSTEM DESCRIPTION}

\section{A. Requirements}

Robotic assistants developed for percutaneous needle insertions [18], [19], [20], [21] often use rigid structures and target static tumors (prostate, breast, neurological targets, ,...). These mechanisms are not adapted for interventions on organs subjected to disturbances related to physiological motions, such as the liver. To perform a liver biopsy for instance, a needle is inserted in the abdomen. This procedure requires to define a fixed needle insertion point as well as a correct orientation of the needle before its insertion. A maximum needle orientation of $45^{\circ}$ around the insertion point could be necessary to reach the target [22], [23]. Moreover, the procedure requires to hold stiffly the needle during the insertion (patient in apnea) so as to ensure precision. It requires also to release the needle, which moves freely with the organ in movement when the patient breathes. This allows to avoid organ laceration. To perform this gesture using a robotic assistance, a mechanism with a minimum 2 rotational DOF is required to perform the needle orientation. A system with RCM corresponding to the insertion point is also necessary. Lastly, the mechanism should allow stiffness modulation in order to avoid organ laceration.

\section{B. System description}

The system, as illustrated in Fig 1, is a variable stiffness 2-DOF tensegrity mechanism with a RCM. It is is composed of two equilateral parallelograms placed at $90^{\circ}$ to each other. The upper bars of the two parallelograms are linked to the needle guide using a sliding pivot. Moreover, the two parallelograms are elevated with an angle $\alpha=15^{\circ}$ with respect to the horizontal plane so as to allow the definition of a common point between the RCM and the needle insertion point. The way of performing the RCM is inspired from [24] with a modification of the way of actuation. Instead of actuating each parallelogram by one actuator, a redundancy of actuation is performed so as to allow stiffness modulation. Each parallelogram is therefore actuated through a pair of cables. Each cable is attached to a corner of the parallelogram at one end and to a spring before rolling up on the actuated pulley on the other end. The mechanism is hence actuated by 4 rotary actuators and has 2 mobilities which are the two orientations. The 2 degrees of kinematic redundancy exploit the prestress property of the tensegrity structures in order to modulate the stiffness of the mechanism [25]. The orientation of the parallelograms are measured using an optical encoder, as shown in Fig 1. A magnetic tracker is also placed inside the needle guide in order to validate the needle guide orientation.

\section{KINEMATIC MODELS}

The center of the reference frame $\mathcal{R}_{0}=\left(O, \mathbf{x}_{0}, \mathbf{y}_{0}, \mathbf{z}_{0}\right)$ is defined as the mechanism RCM. $\mathcal{R}_{1}=\left(O, \mathbf{x}_{1}, \mathbf{y}_{1}, \mathbf{z}_{1}\right)$ is a fixed frame defined such that ${ }^{0} \mathbf{R}_{1}=\operatorname{rot}\left(\mathbf{y}_{0},-\alpha\right.$ ) (cf. Fig IIIa). The fixed frame $\mathcal{R}_{1^{\prime}}=\left(O, \mathbf{x}_{1^{\prime}}, \mathbf{y}_{1^{\prime}}, \mathbf{z}_{1^{\prime}}\right)$ is defined such that $\mathbf{x}_{1^{\prime}}$ and $\mathbf{z}_{1^{\prime}}$ axes are in the plane $\left(\mathbf{y}_{0} \mathbf{z}_{0}\right)$ and that the angle between $\mathbf{x}_{1^{\prime}}$ and $\mathbf{y}_{0}$ is equal to $\alpha$. The fixed frames

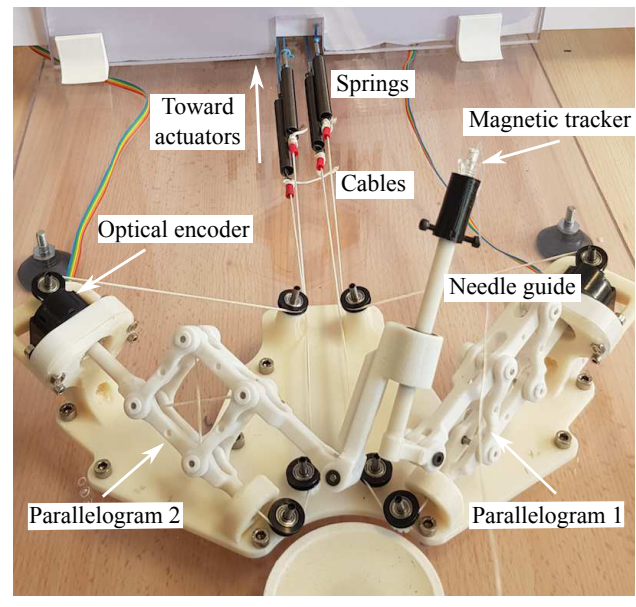

Fig. 1. System overview.

$\mathcal{R}_{2}=\left(O, \mathbf{x}_{2}, \mathbf{y}_{2}, \mathbf{z}_{2}\right)$ and $\mathcal{R}_{2^{\prime}}=\left(O, \mathbf{x}_{2^{\prime}}, \mathbf{y}_{2^{\prime}}, \mathbf{z}_{2^{\prime}}\right)$ are defined respectively such that ${ }^{1} \mathbf{R}_{2}=\operatorname{rot}\left(\mathbf{x}_{1}, \beta_{0}\right)$ and ${ }^{{ }^{\prime}} \mathbf{R}_{2^{\prime}}=$ $\operatorname{rot}\left(\mathbf{x}_{1^{\prime}},-\beta_{0}\right) . \mathbf{z}_{2}$ and $\mathbf{z}_{2^{\prime}}$ axes are coincident and are defined as the common normal to $\mathbf{x}_{1}$ and $\mathbf{x}_{1^{\prime}}$ axes. The angle between $\mathbf{x}_{1}$ and $\mathbf{x}_{1^{\prime}}$ is denoted $\delta . \mathcal{R}_{3}=\left(O, \mathbf{x}_{3}, \mathbf{y}_{3}, \mathbf{z}_{3}\right)$ is a mobile frame attached to the first parallelogram and is defined such that ${ }^{1} \mathbf{R}_{3}=\operatorname{rot}\left(\mathbf{x}_{1}, \beta_{1}\right)$. The same definition applies for $\mathcal{R}_{3^{\prime}}=\left(O, \mathbf{x}_{3^{\prime}}, \mathbf{y}_{3^{\prime}}, \mathbf{z}_{3^{\prime}}\right)$, a mobile frame attached to the second parallelogram: ${ }^{1} \mathbf{R}_{3^{\prime}}=\operatorname{rot}\left(\mathbf{x}_{1^{\prime}}, \beta_{2}\right)$.

Finally, the mobile frame $\mathcal{R}_{n}=\left(O, \mathbf{x}_{n}, \mathbf{y}_{n}, \mathbf{z}_{n}\right)$ is attached to the needle guide. $\mathbf{z}_{n}$ axis is defined along the needle axis. The orientation of $\mathcal{R}_{n}$ with respect to $\mathcal{R}_{3}$ is ${ }^{3} \mathbf{R}_{n}=\operatorname{rot}\left(\mathbf{y}_{3}, \phi_{1}\right)$, with $\phi_{1}$ the angle between $\mathbf{z}_{3}$ and $\mathbf{z}_{n}$ axes, as illustrated in Fig IIIb. The same definition applies for $\mathcal{R}_{3^{\prime}}$ such that ${ }^{3} \mathbf{R}_{n}=\operatorname{rot}\left(\mathbf{y}_{3^{\prime}}, \phi_{2}\right)$.

\section{A. Direct kinematic model}

The direct kinematic model allows to express the orientation of the needle guide, defined by $\mathbf{X}=[\eta \mu 0]^{T}$, according to the joint variables $\boldsymbol{\beta}=\left[\beta_{1} \beta_{2}\right]^{T}$, as illustrated in Fig III (b) and (c). To do this, the orientation of the frame $\mathcal{R}_{n}$ is expressed in the reference frame $\mathcal{R}_{0}$ :

$$
\begin{aligned}
{ }^{0} \mathbf{R}_{n} & ={ }^{0} \mathbf{R}_{1}{ }^{1} \mathbf{R}_{3}{ }^{3} \mathbf{R}_{n} \\
& =\operatorname{rot}\left(\mathbf{y}_{0},-\alpha\right) \operatorname{rot}\left(\mathbf{x}_{1}, \beta_{1}\right) \operatorname{rot}\left(\mathbf{y}_{3}, \phi_{1}\right)
\end{aligned}
$$

The angle $\phi_{1}$ can be computed by solving the closure equation [24]:

$$
\begin{aligned}
{ }^{2} \mathbf{R}_{1}{ }^{1} \mathbf{R}_{3}{ }^{3} \mathbf{R}_{n} \mathbf{z} & ={ }^{2} \mathbf{R}_{2^{\prime}}{ }^{2} \mathbf{R}_{1^{\prime}}{ }^{1}{ }^{\prime} \mathbf{R}_{3^{\prime}}{ }^{3} \mathbf{R}_{n} \mathbf{z} \\
\operatorname{rot}\left(\mathbf{x}_{1}, \beta_{1}^{\prime}\right) \operatorname{rot}\left(\mathbf{y}_{3}, \phi_{1}\right) \mathbf{z} & =\operatorname{rot}\left(\mathbf{z}_{2}, \delta\right) \operatorname{rot}\left(\mathbf{x}_{1^{\prime}}, \beta_{2}^{\prime}\right) \operatorname{rot}\left(\mathbf{y}_{3^{\prime}}, \phi_{2}\right) \mathbf{z}
\end{aligned}
$$

The expression of $\phi_{1}$ is therefore:

$$
\phi_{1}=\operatorname{atan} 2\left(-\sin \left(\beta_{1}^{\prime}\right) \cos (\delta)+\tan \left(\beta_{2}^{\prime}\right) \cos \left(\beta_{1}^{\prime}\right), \sin (\delta)\right)
$$

with: $\beta_{1}^{\prime}=\beta_{1}-\beta_{0}$ and $\beta_{2}^{\prime}=\beta_{2}+\beta_{0}$.

The result of (1) is expressed in the form of:

$$
{ }^{0} \mathbf{R}_{n}=\left[\begin{array}{ccc}
n_{x} & o_{x} & a_{x} \\
n_{y} & o_{y} & a_{y} \\
n_{z} & o_{z} & a_{z}
\end{array}\right]
$$




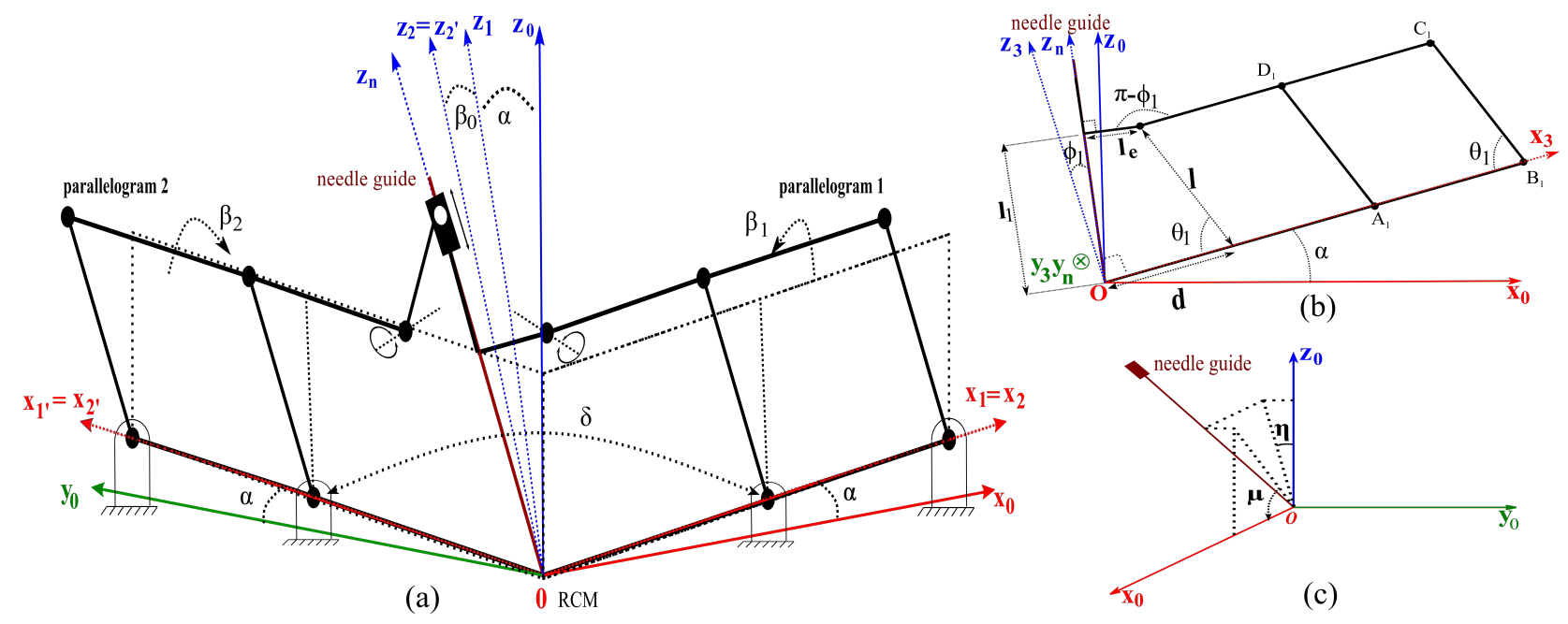

Fig. 2. (a) Schematic of the robot with the frames placement and principals angles ; (b) Planar view of the first parallelogram of the mechanism ; (c) Definition of the orientation of the needle guide.

The orientation of the needle guide is finally computed as:

$$
\begin{aligned}
& \eta=\operatorname{atan} 2\left(-a_{y}, a_{z}\right) \\
& \mu=\operatorname{atan} 2\left(a_{x}, a_{z}\right)
\end{aligned}
$$

\section{B. Inverse kinematic model}

In the inverse kinematic model, the orientation of the needle guide, defined by (4), is supposed to be known. The objective is to determine the joint variables $\beta_{1}$ and $\beta_{2}$. To do so, the equation (1) is pre-multiplied by $\operatorname{rot}\left(\mathbf{x}_{1},-\beta_{1}\right) \operatorname{rot}\left(\mathbf{y}_{0}, \alpha\right)$. From the resulting second row, third column and by replacing $-a_{y} / a_{z}=\tan (\eta)$ and $a_{x} / a_{z}=\tan (\mu)$, we can compute $\beta_{1}$ :

$$
\beta_{1}=\operatorname{atan} 2(\tan (\eta),-\sin (\alpha) \tan (\mu)+\cos (\alpha))
$$

The angle $\phi_{1}$ is computed by considering the first row, third column and third row, third column elements:

$$
\phi_{1}=\operatorname{atan} 2\left(\begin{array}{c}
\cos \left(\beta_{1}\right)(\cos (\alpha) \tan (\mu)+\sin (\alpha)), \\
-\sin (\alpha) \tan (\mu)+\cos (\alpha)
\end{array}\right)
$$

The angles $\beta_{2}^{\prime}$ and $\phi_{2}$ are determined by considering the closure equation (2):

$$
\begin{aligned}
& \beta_{2}^{\prime}=\operatorname{atan} 2\left(\sin (\delta) \tan \left(\phi_{1}\right)+\cos (\delta) \sin \left(\beta_{1}^{\prime}\right), \cos \left(\beta_{1}^{\prime}\right)\right) \\
& \phi_{2}=\operatorname{atan} 2\left(\begin{array}{c}
\cos (\delta) \sin \left(\phi_{1}\right)-\sin (\delta) \sin \left(\beta_{1}^{\prime}\right) \cos \left(\phi_{1}\right), \\
\cos \left(\beta_{1}^{\prime}\right) \cos \left(\phi_{1}\right) / \cos \left(\beta_{2}^{\prime}\right)
\end{array}\right)
\end{aligned}
$$

\section{Workspace and singularities points}

This mechanism is designed to reach all the points of a cone of $45^{\circ}$ around the RCM, so as to satisfy the needle orientation requirements for a liver biopsy procedure. All the geometric parameters are chosen so as to satisfy this workspace, as shown in the Fig 3.

This mechanism has three different regions of singularities. The first one is produced when $\theta_{1}=0$. In this case, the parallelogram 1 is completely leaning forward and lines up with $\mathbf{x}_{1}$-axis, which means the losing of one DOF in the mechanism. The second region of singularity is defined by

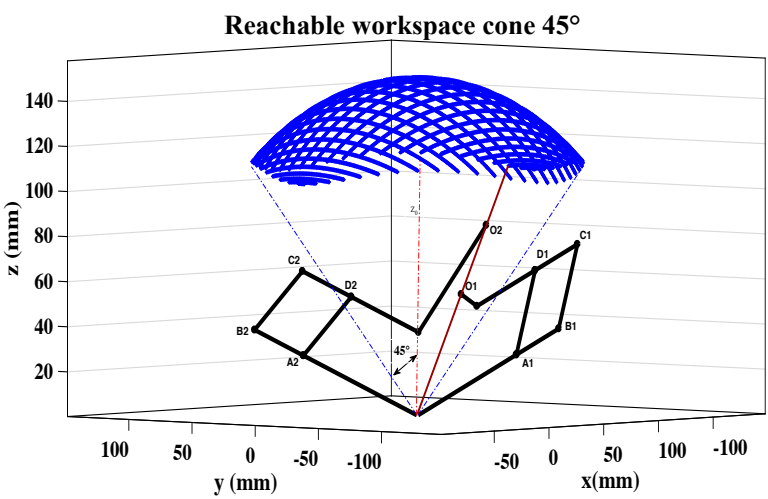

Fig. 3. Reachable workspace of the mechanism.

$\theta_{2}=0$, that produces a similar effect. The third region is determined when the needle guide lies up in the plane $\left(\mathbf{x}_{0} \mathbf{y}_{0}\right)$. All these regions of singularities are outside of the reachable workspace.

\section{STATIC AND STIFFNESS MODELS}

\section{A. Static model}

As aforementioned, the actuation of each parallelogram $i$ is performed using two cables attached to the parallelogram corners $D_{i}$ and $C_{i}$ (cf. Fig 4). These cables are attached at the other end to springs of stiffness $k$ before rolling up on actuators. The vector angular position of these actuators is denoted $\boldsymbol{\alpha}=\left[\begin{array}{llll}\alpha_{11} & \alpha_{21} & \alpha_{12} & \alpha_{22}\end{array}\right]^{T}$. The tensions in the cables applied to the parallelogram $i$ are denoted $\boldsymbol{\tau}_{i}=\left[\begin{array}{ll}\tau_{1 i} & \tau_{2 i}\end{array}\right]^{T}$. The static of the parallelogram $i$ can be described as:

$$
\mathbf{W}_{i} \boldsymbol{\tau}_{i}=\Gamma_{i}
$$

where $\mathbf{W}_{i}=\left[l \sin \left(\theta_{i} / 2\right) l \cos \left(\theta_{i} / 2\right)\right]$ and $\Gamma_{i}$ being the torque generated by the cables on the bar $A_{i} D_{i}$. The angle $\theta_{i}$ can 


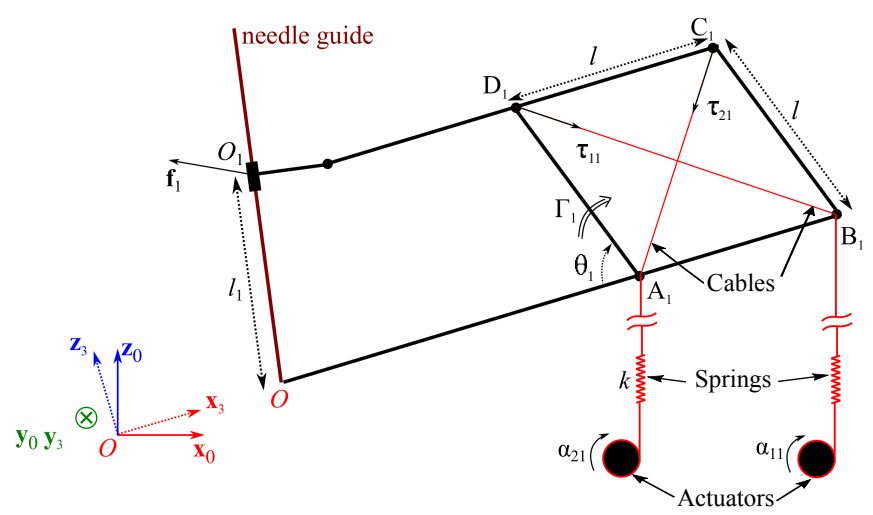

Fig. 4. Overview of the forces applied to the first parallelogram.

be computed by considering the closure form (cf. Fig III(b)):

$$
\mathbf{d}+\mathbf{l}+\mathbf{l}_{e}+\mathbf{l}_{i}=\mathbf{0}
$$

$\theta_{1}$ can be computed by projecting (11) along $\mathbf{x}_{3}$ and $\mathbf{z}_{3}$ axes, we obtain:

$$
\left\{\begin{array}{r}
d-l \cos \left(\theta_{1}\right)-l_{e} \cos \left(\phi_{1}\right)-l_{1} \sin \left(\phi_{1}\right)=0 \\
l \sin \left(\theta_{1}\right)+l_{e} \sin \left(\phi_{1}\right)-l_{1} \cos \left(\phi_{1}\right)=0
\end{array}\right.
$$

This system of equations can be solved by multiplying the first equation by $\cos \left(\phi_{1}\right)$ and the second equation by $\sin \left(\phi_{1}\right)$. Subtracting the obtained equations which leads to:

$$
d \cos \left(\phi_{1}\right)-l_{e}-l \cos \left(\phi_{1}\right) \cos \left(\theta_{1}\right)-l \sin \left(\phi_{1}\right) \sin \left(\theta_{1}\right)=0
$$

Using an analogical reasoning although by projecting (11) along $\mathbf{x}_{3^{\prime}}$ and $\mathbf{z}_{3^{\prime}}$ axes, $\theta_{2}$ can be obtained. The angle $\theta_{i}$ for the parallelogram $i$ can thus be computed as:

$$
\theta_{i}=\operatorname{atan} 2\left(\begin{array}{c}
\left(x z+y \sqrt{x^{2}+y^{2}-z^{2}}\right) / l^{2}, \\
\left(y z-x \sqrt{x^{2}+y^{2}-z^{2}}\right) / l^{2}
\end{array}\right)
$$

with: $x=l \cos \left(\phi_{i}\right), y=l \sin \left(\phi_{i}\right)$ and $z=d \cos \left(\phi_{i}\right)-l_{e}$

\section{B. Stiffness model}

To elaborate the stiffness model, one should compute the force contribution, generated by the tensions in the cables, of each parallelogram to the needle guide. These forces are represented in the needle guide frame $\mathcal{R}_{n}$ and computed by applying the principle of virtual works in each bar of the parallelogram. The contact point between the needle guide and the parallelogram $i$ is denoted $O_{i}$ and the force applied at this point is defined as $\mathbf{f}_{i}$ (cf. Fig 4). These forces are expressed first in the frame attached to each parallelogram. $\mathbf{f}_{i}$,expressed in $\mathcal{R}_{3}$, gives :

$$
\left\{\begin{array}{l}
{ }^{3} f_{1 x}=\frac{-\tau_{11} \sin \left(\theta_{1} / 2\right)+\tau_{21} \cos \left(\theta_{1} / 2\right)}{\sin \left(\theta_{1}\right)-\tan \left(\phi_{1}\right) \cos \left(\theta_{1}\right)} \\
{ }^{3} f_{1 y}=0 \\
{ }^{3} f_{1 z}=\frac{-\tau_{11} \sin \left(\theta_{1} / 2\right)+\tau_{21} \cos \left(\theta_{1} / 2\right)}{\cos \left(\theta_{1}\right)-\cot \left(\phi_{1}\right) \sin \left(\theta_{1}\right)}
\end{array}\right.
$$

This force ${ }^{3} \mathbf{f}_{1}$ can be expressed in the frame $\mathcal{R}_{n}$ as:

$$
{ }^{n} \mathbf{f}_{1}={ }^{n} \mathbf{R}_{3}{ }^{3} \mathbf{f}_{1}=\operatorname{rot}\left(\mathbf{y}_{3},-\phi_{1}\right)^{3} \mathbf{f}_{1}
$$

After simplification by using trigonometric relations, the expression of ${ }^{n} \mathbf{f}_{1}$ is defined as:

$$
\left\{\begin{aligned}
{ }^{n} f_{1 x} & =\frac{-\tau_{11} \sin \left(\theta_{1} / 2\right)+\tau_{21} \cos \left(\theta_{1} / 2\right)}{-\sin \left(\phi_{1}\right) \cos \left(\theta_{1}\right)+\sin \left(\theta_{1}\right) \cos \left(\phi_{1}\right)} \\
{ }^{n} f_{1 y} & =0 \\
{ }^{n} f_{1 z} & =0
\end{aligned}\right.
$$

Using a similar reasoning, the expression of ${ }^{n} \mathbf{f}_{2}=$ $\left.{ }^{n} f_{2 x}{ }^{n} f_{2 y}{ }^{n} f_{2 y}\right]$ could be obtained.

At the equilibrium configuration, the sum of the torques applied to the needle guide must be equal to zero:

$$
\boldsymbol{\Gamma}_{e}+\mathbf{l}_{1} \times{ }^{n} \mathbf{f}_{1}+\mathbf{l}_{2} \times{ }^{n} \mathbf{f}_{2}=\mathbf{0}
$$

with $\Gamma_{e}$ being the external torque, that is equal to:

$$
\boldsymbol{\Gamma}_{e}=\left(\begin{array}{lll}
{ }^{n} f_{2 y} \cdot l_{2} & -{ }^{n} f_{1 x} \cdot l_{1} & 0
\end{array}\right)^{T}
$$

with $l_{i}=\sqrt{d^{2}+l^{2}+2 \cdot d \cdot l \cdot \cos \left(\theta_{i}\right)-l_{e}^{2}}$ being the distance between $O_{i}$ and the RCM (cf. Fig 4).

The stiffness of the needle guide is then obtained by:

$$
\begin{aligned}
\mathbf{K} & =-\frac{d \boldsymbol{\Gamma}_{e}}{d \mathbf{X}^{T}} \\
& =\left(\begin{array}{lll}
k_{\eta} & k_{\mu} & 0
\end{array}\right)^{T}
\end{aligned}
$$

where $k_{\eta}$ and $k_{\mu}$ represent the angular stiffnesses of the needle guide around the mobilities of the mechanism.

For ease of notation, let's define $\Gamma_{e y}={ }^{n} f_{1 x} \cdot l_{1}$. The stiffness $k_{\mu}$ is computed as:

$$
k_{\mu}=\frac{d \Gamma_{e y}}{d \mu}=\frac{\partial \Gamma_{e y}}{\partial \theta_{1}} \frac{\partial \theta_{1}}{\partial \mu}+\frac{\partial \Gamma_{e y}}{\partial \phi_{1}} \frac{\partial \phi_{1}}{\partial \mu}
$$

with $\frac{\partial \theta_{1}}{\partial \mu}=\frac{\partial \theta_{1}}{\partial \phi_{1}} \frac{\partial \phi_{1}}{\partial \mu}$. This can be calculated using (7) and (14). The expression of $k_{\mu}$ is reformulated as:

$$
k_{\mu}=\left(\frac{\partial \Gamma_{e y}}{\partial \theta_{1}} \frac{\partial \theta_{1}}{\partial \phi_{1}}+\frac{\partial \Gamma_{e y}}{\partial \phi_{1}}\right) \frac{\partial \phi_{1}}{\partial \mu}
$$

The computation of $k_{\eta}$ can be obtained using the same reasoning.

\section{CONTROL}

\section{A. Control Principle}

The main objective is to control asynchronously the position and the stiffness of the mechanism. The control strategy (cf. Fig 5) is based on a tension distribution algorithm, inspired from [26]. This algorithm, as shown below, allows the stiffness modulation. The control strategy has an external loop to control the configuration of the parallelograms defined by $\boldsymbol{\theta}=\left[\begin{array}{ll}\theta_{1} & \theta_{2}\end{array}\right]^{T}$ with respect to the reference $\boldsymbol{\theta}^{d}=\left[\begin{array}{ll}\theta_{1}^{d} & \theta_{2}^{d}\end{array}\right]^{T}$. This reference can be computed from the desired orientation $\mathbf{X}^{d}=\left[\begin{array}{lll}\eta^{d} & \mu^{d} 0\end{array}\right]^{T}$ of the needle guide using the equations of the inverse kinematic model and (14).

The error of the external loop is multiplied by a proportional controller $\mathbf{C}_{\boldsymbol{\theta}}$ that generates a virtual torque $\boldsymbol{\Gamma}^{v}=\left[\begin{array}{ll}\Gamma_{1}^{v} & \Gamma_{2}^{v}\end{array}\right]^{T} . \boldsymbol{\Gamma}^{v}$ and $\boldsymbol{\theta}$ are used as inputs to the tension distribution algorithm.

The output of this algorithm is a reference vector tension $\boldsymbol{\tau}^{d}=\left[\begin{array}{ll}\boldsymbol{\tau}_{1}^{d} & \boldsymbol{\tau}_{2}^{d}\end{array}\right]^{T}$. The vector $\boldsymbol{\tau}_{i}^{d}=\left[\begin{array}{ll}\tau_{1 i}^{d} & \tau_{2 i}^{d}\end{array}\right]^{T}$ contains 


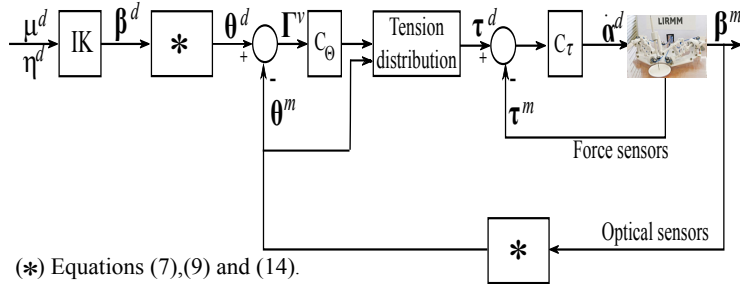

Fig. 5. Position/stiffness control strategy.

the tensions in the cables that move the parallelogram $i$. These tensions are supposed to generate the virtual torque $\Gamma_{i}^{v}$ that should be applied to the parallelogram $i$. Besides, the algorithm has to ensure that the vector $\tau_{i}^{d}$ remains always positive and bounded in a defined interval $\left[\boldsymbol{\tau}_{\min }, \boldsymbol{\tau}_{\max }\right]$ for all the possible configurations of the mechanism. These bounds are defined as $\boldsymbol{\tau}_{\min }=\left[\begin{array}{ll}\tau_{\min } & \tau_{\min }\end{array}\right]^{T}$ and $\boldsymbol{\tau}_{\max }=$ $\left[\begin{array}{ll}\tau_{\max } & \tau_{\max }\end{array}\right]^{T}$. The tension distribution algorithm solves:

$$
\mathbf{W}_{i} \boldsymbol{\tau}_{i}^{d}=\Gamma_{i}^{v} \text { with } \boldsymbol{\tau}_{\min } \leq \boldsymbol{\tau}_{i}^{d} \leq \boldsymbol{\tau}_{\max }
$$

The solution to (23) has the form [27]:

$$
\boldsymbol{\tau}_{i}^{d}=\mathbf{W}_{i}^{+} \Gamma_{i}^{v}+\mathbf{N}_{i} \lambda_{i}
$$

$\mathbf{W}_{i}^{+}$denotes the Moore-Penrose pseudoinverse of $\mathbf{W}_{i}$, $\mathbf{N}_{i}=\left[-l \cos \left(\theta_{i} / 2\right)-l \sin \left(\theta_{i} / 2\right)\right]^{T}$ a basis of $\mathbf{W}_{i}$ null space. $\lambda_{i}$ is a scalar to be defined that satisfies (23). The bounds of $\lambda_{i}$ can be obtained by solving the inequality system:

$$
\boldsymbol{\tau}_{\text {min }}-\mathbf{W}_{i}^{+} \Gamma_{i}^{v} \leq \mathbf{N}_{i} \lambda_{i} \leq \boldsymbol{\tau}_{\max }-\mathbf{W}_{i}^{+} \Gamma_{i}^{v}
$$

These bounds are defined as $\left[\lambda_{\text {min }}^{i}, \lambda_{\text {max }}^{i}\right]$ for each parallelogram. They are variable and depend on the configuration of the mechanism as well the tensions limit. $\lambda_{i}$ must be chosen inside this interval so as to satisfy (23).

Once $\tau^{d}$ is computed using (24), it is compared to $\tau$ that contains the real tensions in the cables. These tensions are measured using force sensors. A tension controller $\mathbf{C}_{\tau}$ is considered to control these tensions. The output of this tension controller is sent to the actuators as desired velocities $\dot{\boldsymbol{\alpha}}^{d}=\left[\begin{array}{llll}\dot{\alpha}_{11}^{d} & \dot{\alpha}_{21}^{d} & \dot{\alpha}_{12}^{d} & \dot{\alpha}_{22}^{d}\end{array}\right]^{T}$. The tension inner loop must have a wider bandwidth compared to the position outer loop in order to ensure stability.

\section{B. Stiffness Modulation}

To simplify the notations in (22) that expresses the angular stiffness $k_{\mu}$ produced by the first parallelogram, let's define: $a_{1}=\frac{\partial \Gamma_{e y}}{\partial \theta_{1}}, a_{2}=\frac{\partial \theta_{1}}{\partial \phi_{1}}, a_{3}=\frac{\partial \Gamma_{e y}}{\partial \phi_{1}}$ and $a_{4}=\frac{\partial \phi_{1}}{\partial \mu}$. which yields:

$$
k_{\mu}=\left(a_{1} a_{2}+a_{3}\right) a_{4}
$$

Assuming that the system is in an equilibrium configuration $\Gamma^{v}=\mathbf{0}$ and that the desired tensions are reached $\left(\boldsymbol{\tau}=\boldsymbol{\tau}^{d}\right)$, the equation (24) for the parallelogram 1 becomes:

$$
\tau_{1}=\mathbf{N}_{1} \lambda_{1}
$$

It can be shown that, at the equilibrium configuration, $a_{1}=-\frac{l_{1}}{2 \sin \left(\theta_{1}+\phi_{1}\right)} \boldsymbol{\tau}_{1}^{T} \mathbf{N}_{1}$ and $a_{3}=0$. Hence:

$$
k_{\mu}=-a_{2} a_{4} \frac{l_{1}}{2 \sin \left(\theta_{1}+\phi_{1}\right)} \boldsymbol{\tau}_{1}^{T} \mathbf{N}_{1}
$$

Pre-multiplying (27) by $\mathbf{N}_{1}^{T}$ yields:

$$
\mathbf{N}_{1}^{T} \boldsymbol{\tau}_{1}=\mathbf{N}_{1}^{T} \mathbf{N}_{1} \lambda_{1}
$$

Substituting (29) in (28) leads to:

$$
k_{\mu}=-\frac{l_{1} a_{2} a_{4} l^{2} \lambda_{1}}{2 \sin \left(\theta_{1}+\phi_{1}\right)}
$$

The relationship between $k_{\eta}$ and $\lambda_{2}$ can be established by applying similar reasoning. Through this result, it is therefore possible to modulate explicitly the stiffness of the mechanism by varying $\lambda_{i}$. It is also important to highlight that it is possible to modulate stiffness only along one mobility of the needle guide without affecting the other mobility.

\section{Setup and experimentation}

In order to validate the control strategy proposed in the previous subsection, a prototype of the mechanism is developed, as shown in Fig 1, and an experiment is conducted. The mechanism is actuated using 4 DC motors, two for each parallelogram. Polyethylene fibre cables are deployed for motion transmission. 4 force sensors are used to measure the cable tensions. Each sensor is placed between one motor and a spring. Moreover, two optical encoders are used to measure the angles $\beta_{1}$ and $\beta_{2}$. Finally, a magnetic tracker with a resolution of $0.5^{\circ}$ is placed inside the needle guide in order to validate the needle guide orientation obtained using the developed kinematic models.

The experiment is divided into two phases. In the first phase, $t \in[0,20] s$, the position tracking performance is evaluated. In the second phase, $t \in[20,40] s$, the stiffness modulation is assessed. During the first phase, a fifth-order polynomial equation is used to generate the cartesian trajectory along the two mobilities: $\eta^{d}$ from $0^{\circ}$ to $-20^{\circ}$ and $\mu^{d}$ from $0^{\circ}$ to $20^{\circ}$. The admissible tensions in the cables are set between $\tau_{\min }=4 N$ and $\tau_{\max }=20 N$. Finally, the values of $\lambda_{1}$ and $\lambda_{2}$ are chosen respectively at their lower admissible values $\lambda_{\min }^{1}$ and $\lambda_{\min }^{2}$ so as to reduce the transversal forces, $\mathbf{f}_{1}$ and $\mathbf{f}_{2}$, applied to the needle guide during the positioning. During the second phase, a linear variation of $\lambda_{1}$ and $\lambda_{2}$ is performed, passing from $\lambda_{\text {min }}^{i}$ to $\lambda_{\text {max }}^{i}$ for each parallelogram $i$. This variation allows to evaluate the capacity to modulate the angular stiffness of the needle guide.

\section{Results}

Three different curves can be observed in Fig 6 (ab). These curves correspond to the desired angles $\left({ }^{d}\right)$, the estimated $\left({ }^{e}\right)$ ones using the optical encoders as well as the direct kinematic model, and finally the measured ones $\left({ }^{m}\right)$ using the magnetic tracker. During the position tracking (Fig 6 , a-b), a dead-zone of about $6 s$, caused by static frictions, is observed. After this zone, the tracking improves with a 

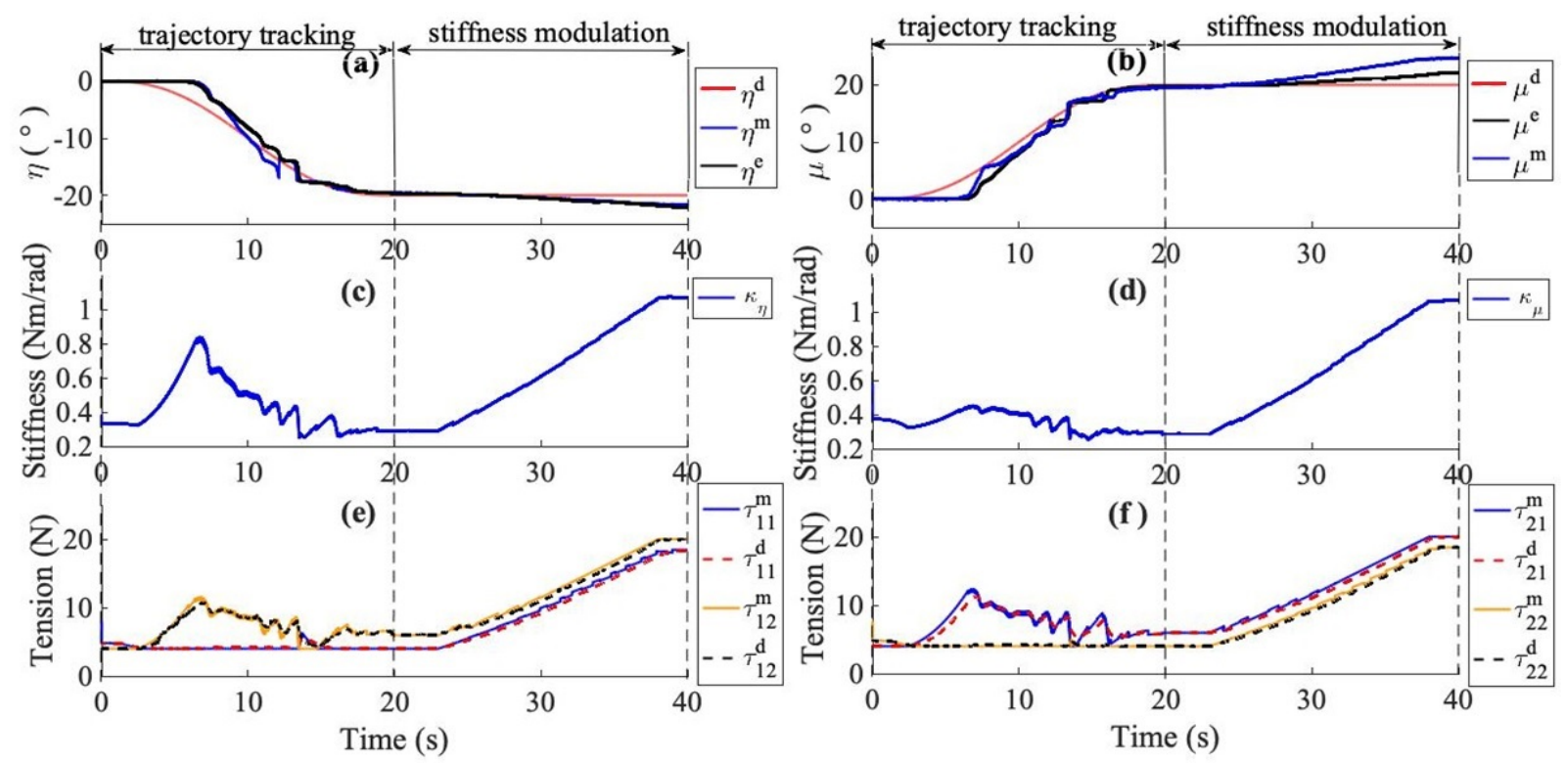

Fig. 6. Tracking trajectory and stiffness modulation: Left $\eta$ variation and first parallelogram results and Right $\mu$ variation and second parallelogram results

root mean square error, respectively, of $1.41^{\circ}$ between $\eta^{d}$ and $\eta^{e}$, and of $1.47^{\circ}$ between $\mu^{d}$ and $\mu^{e}$. The static error in both trajectories is less than $2 \%$. Besides, a good tension tracking is observed during the two phases, as seen in Fig 6 (e-f). The robustness of the control strategy with respect to the uncertainty of the kinematic parameters is also analyzed. A variation of ${ }_{-}^{+} 10 \%$ of these parameters, does not present changes in the position static error. Moreover, the root mean square error of the tracking is less than $0.1^{\circ}$ compared to the results obtained without parameters variation.

In order to validate the models of the mechanism, a comparison between the estimated results and the measured ones is realized. A root mean square error of about $0.8^{\circ}$ along $\mu$ and $\eta$ directions is observed, knowing that the precision of the tracker is $0.5^{\circ}$. This error could be explained by the use of a rigid model in the estimation of the needle guide orientation, whereas the prototype is 3D printed, therefore presents some flexibilities. The mechanical backlashes have also an impact on this error. Besides and as it can be observed in the results, a stick and slip phenomenon occurs during the position tracking. This phenomenon is mainly caused by static frictions. A mechanical improvement of the sliding joint would reduce this frictions and attenuate the dead zone present at the beginning of the trajectories.

Regarding the stiffness modulation, by varying $\lambda_{1}$ and $\lambda_{2}$ the angular stiffnesses, $k_{\eta}$ and $k_{\mu}$, vary gradually as seen in (Fig 6, c-d). The stiffnesses variation is consistent with the cable tensions variation, as it can be observed in Fig 6 (e-f). A maximal angular stiffness of $1.06 \mathrm{Nm} / \mathrm{rad}$ is reached along the two mobilities. Theoretically, no motions of the mechanism should occur during the stiffness modulation. However, a $10 \%$ position error is observed. This can be explained by the increasing forces $\mathbf{f}_{1}$ and $\mathbf{f}_{2}$, that are due to the cable tensions augmentation.Indeed, these forces produce a small deflection in the sliding joint, given its flexibility. A rigidity improvement of the sliding joint should reduce this orientation error.

\section{CONCLUSION}

In this paper, a 2-DOF spatial remote center of motion cable-driven mechanism is proposed. Based on a tensegrity system, this mechanism allows to control asynchronously the reconfiguration while modulating explicitly its stiffness. The stiffness model is derived analytically and linked to the tension distribution algorithm for a decoupled stiffness modulation.

Trajectory tracking is assessed on the developed prototype obtaining a static error less than $2 \%$ between the estimated and reference orientations. An evaluation of the position tracking has been performed using a magnetic tracker. The results show that the root mean square error, between the estimated orientation and the measured one is about $0.8^{\circ}$, knowing that the resolution of the magnetic tracker is $0.5^{\circ}$.

The experiment has shown also that it is possible to explicitly modify the stiffness of the mechanism by a factor of 3.5. A position static error of $10 \%$ has been observed during the stiffness modulation. To our sense, these results are satisfactory, promising and show that it is possible to vary the stiffness of the needle guide without affecting roughly its position.

Future work will be focused in the mechanical improvement of the mechanism, particularly in the re-design of the sliding joint. New control strategies based on friction compensation would be of interest to reduce the stick and slip phenomenon and the dead zone observed in the trajectory tracking. An Interesting perspective would be the implementation of a set up, with sensors placed in the parallelograms, in order to validate the stiffness modulation of the system. 


\section{REFERENCES}

[1] B. Fuller : Tensile-integrity structures, United States Patent No. 3,063,521, 13 novembre 1962 .

[2] A. Pugh, "An introduction to tensegrity. Berkeley", University of California Press, Los Angeles, Californie, United States, first edition, 1976.

[3] K. Snelson, "Continuous tension, discontinuous compression structures", United States Patent No. 3,169,611,1965.

[4] R. Motro, "Tensegrity: Structural Systems for the Future", first edition, Elsevier Science, 2003.

[5] C. Paul, F. J. Valero-Cuevas and H. Lip-Son, "Design and control of tensegrity robots for locomotion", IEEE Trans. Robot., pp. 944-957, vol. 22, no 5, 2006.

[6] J. P. Pinaud, S. Solari and R. E. Skelton, "Deployment of a Class 2 Tensegrity Boom", in SPIE's 11th Annual International Symposium on Smart Structures and Materials, 2004.

[7] M. Masic and R. E. Skelton, "Open-loop control of class-2 tensegrity towers", vol. 5383, pp. 298, 2004.

[8] R. E. Skelton and M. C. De Oliveira, "Tensegrity Systems", Springer, 2009.

[9] C. Sultan, "Modeling, design, and control of tensegrity structures with applications", Purdue University, 1999.

[10] J. B. Aldrich, and R. E. Skelton, "Time- energy optimal control of hyper-actuated mechanical systems with geometric path constraints", in Proceedings of the 44th IEEE Conference on Decision and Control, pp. 8246-8253, 2005.

[11] C. Sultan, 'Tensegrity Deployment Using Infinitesimal Mechanisms". International Journal of Solids and Structures.2014.

[12] M. Fujiia, S. Yoshiia and Y. Kakazub, "Movement control of tensegrity robot”, Intell. Auton. Syst. 9 IAS-9, vol. 9, pp. 290-297, 2006.

[13] V. Sunspiral, A. Agogino and D. Atkinson, "Super Ball Bot Structures for Planetary Landing and Exploration", NIAC Phase 2, 2015.

[14] N. Kanchanasaratool and D. Williamson, "Modelling and control of class NSP tensegrity structures", Int. J. Control, vol. 75, no 2, pp. 123-139, 2002.

[15] J. Van De Wijdeven and B. De Jager, "Shape change of tensegrity structures : design and control", in Proceedings of the American Control Conference, pp. 2522-2527 vol. 4, 2005.
[16] J. B. Aldrich, R. E. Skelton and K. Kreutz-Delgado, "Control synthesis for a class of light and agile robotic tensegrity structures", in Proceedings of the American Control Conference, vol. 6, pp. 5245-5251 2003.

[17] Q. Boehler, S. Abdelaziz, M. Vedrines, P. Poignet, P. Renaud, "From modeling to control of a variable stiffness device based on a cabledriven tensegrity mechanism", Mechanism and Machine Theory, Elsevier, pp. 1-12, vol. 107, 2017

[18] A. Melzer, B. Gutmann et al. "INNOMOTION for Percutaneous Image-Guided Interventions.", IEEE Engineering in Medicine and Biology Magazine. 2008.

[19] I. Bricault et al., "Light Puncture Robot for CT and MRI Interventions,", IEEE Engineering in Medicine and Biology Magazine, vol. 27, no. 3, pp. 42-50, May-June 2008.

[20] B. Arnaud, F. Geiskopf and Pierre Renaud "Toward Unibody Robotic Structures with Integrated Functions using Multimaterial Additive Manufacturing: Case Study of an MRI-compatible Interventional Device", IEEE/RSJ International Conference on Intelligent Robots and Systems (IROS). 2015.

[21] M. E. M. K. Abdelaziz, V. Groenhuis et al. "Controlling the Stormram 2: An MRI-compatible robotic system for breast biopsy", IEEE International Conference on Robotics and Automation (ICRA). 2017.

[22] J.Kaufman and M.Lee, "Vascular and Interventional Radiology", the Requesites, Mosby Ed. 2004.

[23] C. J. Walsh, N. C. Hanumara, A. H. Slocum, J. - A. Shepard, and R. Gupta, "A Patient-Mounted, Telerobotic Tool for CT-Guided Percutaneous Interventions," ASME Journal of Medical Devices, vol. 2, no. 1, 2008.

[24] R. Baumann, W. Maeder, D. Glauser and R. Clavel, "The PantoScope: a spherical remote-center-of-motion parallel manipulator for force reflection", In: Proceedings of IEEE international conference on robotics and automation, pp. 718-23, 1997.

[25] M. Azadi, S.Behzadipour, "Variable antagonistic stiffness element using tensegrity mechanism", in: Proceedings of the 2007 ASME IMECE 2007, Seattle, WA, USA, 2007, pp. 21-28.

[26] S. Abdelaziz, L. Barbé, P. Renaud and B Bayle, "Control of cabledriven manipulators in the presence of friction", Mechanism and Machine Theory, Elsevier, pp.139-147, vol. 107, 2017.

[27] M. Gouttefarde, J. Lamaury, C. Reichert, T. Bruckmann, ”A Versatile Tension Distribution Algorithm for n-DOF Parallel Robots Driven by n+2 Cables," IEEE Transactions on Robotics, Vol. 31, No. 6, pp. 1444$1457,2015$. 